\title{
Optical coherence structure: A novel tool for light manipulation
}

\author{
CHEN YaHong ${ }^{1} \&$ CAI YangJian ${ }^{1,2 *}$ \\ ${ }^{1}$ Institute of Optics, School of Physical Science and Technology, Soochow University, Suzhou 215006, China; \\ ${ }^{2}$ Shandong Provincial Engineering and Technical Center of Light Manipulation \& Shandong Provincial Key Laboratory of Optics and Photonic Devices, \\ School of Physics and Electronics, Shandong Normal University, Jinan 250014, China
}

Received November 11, 2021; accepted November 16, 2021; published online December 9, 2021

Citation: Chen Y H, Cai Y J. Optical coherence structure: A novel tool for light manipulation. Sci China Tech Sci, 2022, 65: 740-742,

https://doi.org/10.1007/s11431-021-1966-6

Light field manipulation refers to controlling light characteristics in spatial, temporal, frequency, or spatiotemporal domains. The created structured light fields have enabled progress in a broad range of interdisciplinary science and technology $[1,2]$, owing to their novel physical properties. The traditional light manipulation is mainly focused on the government of deterministic features (sometimes called single-point characteristics) of fully coherent light. However, because of the omnipresent uncorrelated spontaneous emission from atoms, all optical fields, whether encountered in nature or created in a laboratory, have more or less random fluctuations within their physical properties. The fluctuations within the single-point characteristics are too rapid to be directly detected. Thus, the statistical approaches must be used to describe the average correlations of the field among pairs of points. The optical coherence is an efficient tool to describe the (multiple-point) statistical property of random light. It has been shown that light field with controlled optical coherence, i.e., partially coherent light field, has advantages in specific applications such as speckle-free imaging [3] due to its low coherence nature.

Typically, the manipulation of optical coherence refers to the light's coherence width control $[4,5]$. Thus, the optical coherence can be viewed as a 'global' feature that shows the ability of the field to form the self-interference pattern. Optical coherence structure-a recently proposed concept that describes the spatial structure of the correlations among the

*Corresponding author (email: yangjiancai@suda.edu.cn) light field-shows, on the other hand, the 'local' statistical property of random light. Nowadays, the spatial coherence structure has been regarded as a novel and powerful tool for manipulating the statistical properties of light fields during propagation or light-matter interactions. It has been shown that the optical beam with engineered spatial coherence structure can display the self-focusing, self-spitting, and self-shaping effects during propagation in free space. Moreover, optical scattering properties can be controlled by engineering the spatial coherence structure of incident light.

At the same, the coherence structure has been viewed as a new degree of freedom for various applications. Peng et al. [6], recently, showed that the coherence structure can be used to realize optical encryption (see Figure 1). Information encryption with the aid of optical technologies has been studied extensively in the past decade due to remarkable multidimensional capabilities and ultrafast modulation speed afforded by the light fields. Like other first-order deterministic field characteristics such as optical phase, intensity, and polarization, the second-order coherence structure can also be viewed as an efficient degree of freedom to carry optical information. There are two advantages of coherence-based encryption. First, the complexity of coherence structure measurement enhances the security of the encryption. Second, the relative insensitivity of the statistical characteristics of light to environmental noise makes the protocol robust against the environmental fluctuations, e.g., robust to the turbulent atmosphere. Such novel coherence-based encryption, thus, can be used in the application scenarios where an attacker aims to 


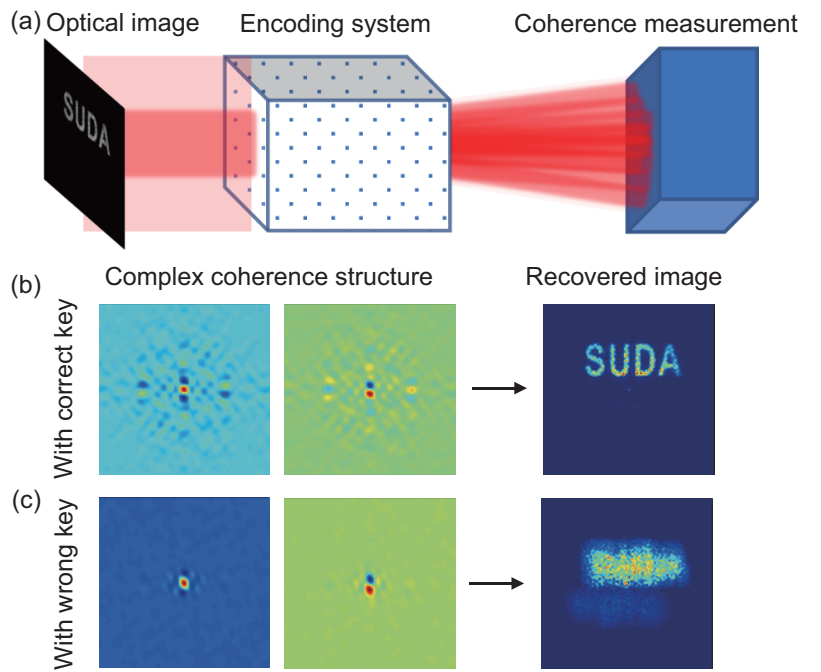

Figure 1 (Color online) Optical encryption based on spatial coherence structure engineering. (a) Optical image is encoded in the spatial coherence structure through a generalized van Cittert-Zernike theorem and is recovered by the coherence measurement; (b) spatial distribution of complex coherence structure and recovered image with the correct encryption key; (c) the results for the case with the wrong encryption key.

deliberately destroy the ciphertext rather than temper it.

Due to the robustness of the second-order coherence structure in a complex environment, the coherence structure engineering can also be used in a robust far-field optical imaging application. In the application, the optical image is firstly encoded in the spatial coherence structure of a partially coherent source through the (generalized) van Cittert-Zernike theorem. By introducing a second-order cross phase into the spatial coherence structure, Liu et al. [7] found that the spatial distribution of the spatial coherence structure in the source plane can be well reconstructed in the far field. Thus, the optical image can be recovered by measuring the complex spatial coherence structure in the far field. Such protocol performs well even when the transmission link is blocked partially by the obstacles or filled with strong atmospheric turbulence. Remarkably, some structured, fully coherent light fields such as diffraction-free beams may also show a similar robust feature in a complex environment. However, when the size of the obstacles is large or the strength of the turbulent atmosphere is strong within the propagation link, the nonnegligible interference effects will distort the far-field imaging quality. The approach based on spatial coherence structure engineering is still robust.

In addition to the robustness in the turbulent atmosphere, the optical coherence structure can also be transferred between the incident and reflected/transmitted fields from a random multiple-scattering medium, which indicates new applications such as imaging through random media with coherence structure engineering. Huang et al. [8] showed that by measuring the complex spatial coherence structure of transmitted light with the generalized Hanbury Brown-Twiss ex- periment, the moving image information, including its shape and position, hidden behind the rotating ground glass disk can be recovered well. The robust feature of the spatial coherence structure can also be used in non-line-of-sight imaging. By measuring the spatial coherence distribution of the reflected light from rough scattering wall, Batarseh et al. [9] showed that the position and the shape of an incoherent object hidden in non-line-of-sight places can be well reconstructed.

As an efficient tool for light manipulation, optical coherence structure has brought in a series of novel optical applications. Compared to the fully coherent methods, the coherence-based approaches have several advantages such as the created beams are robust in a complex environment and have fewer speckles. Up to now, the experiments for optical coherence structure engineering are carried out outside the laser cavity, e.g., with the aid of spatial light modulator and digital micro-mirror device, due to the flexibility of the performance. However, the speed for spatial coherence structure modulation can only reach to $\mathrm{kHz}[10]$ with these methods, which limits the applications where ultra-fast modulation is needed, such as in the free-space optical communication. This limitation could be solved with new photoelectric materials having fast modulation speed. Moreover, in some particular applications where the high-power and lowcoherence light fields are required, such as speckle-free inertial confinement fusion, a realization of the coherence structure engineering inside the laser cavity may be the most efficient approach. This could be achieved with the help of unconventional, complex laser cavities [11]. Furthermore, how to compactly modulate light's coherence structure with flat optical elements such as metasurfaces [12] is a challenge in nanophotonics. Finally, optical coherence structure engineering is focused mainly on the spatial domain. The coherence structure engineering in the temporal or spatiotemporal domain may open new doors for novel applications, e.g., the high-order correlation imaging in the spatiotemporal domain.

This work was supported by the National Key Research and Development Program of China (Grant No. 2019YFA0705000), the National Natural Science Foundation of China (Grant Nos. 91750201, 11974218, 11904247, and 12192254), the Innovation of Group of Jinan (Grant No. 2018GXRC010), the Local Science and Technology Development Project of the Central Government (Grant No. YDZX20203700001766), the China Postdoctoral Science Foundation (Grant No. 2019M661915), and the Natural Science Foundation of the Jiangsu Higher Education Institutions of China (Grant No. 19KJB140017).

1 Forbes A, de Oliveira M, Dennis M R. Structured light. Nat Photon, 2021, 15: 253-262

2 Wang J. Twisted optical communications using orbital angular momentum. Sci China-Phys Mech Astron, 2019, 62: 034201

3 Redding B, Choma M A, Cao H. Speckle-free laser imaging using random laser illumination. Nat Photon, 2012, 6: 355-359

4 Cai Y J, Chen Y H, Yu J Y, et al. Generation of partially coherent beams. Prog Opt, 2017, 62: 157-223

5 Dong M, Zhao C L, Cai Y J, et al. Partially coherent vortex beams: 
Fundamentals and applications. Sci China-Phys Mech Astron, 2021, 64: 224201

6 Peng D M, Huang Z F, Liu Y L, et al. Optical coherence encryption with structured random light. PhotoniX, 2021, 2: 6

7 Liu Y, Chen Y, Wang F, et al. Robust far-field imaging by spatial coherence engineering. Opto Electron Adv, 2021, 4: 210027

8 Huang Z, Chen Y, Wang F, et al. Measuring complex degree of coherence of random light fields with generalized Hanbury Brown-Twiss experiment. Phys Rev Appl, 2020, 13: 044042
9 Batarseh M, Sukhov S, Shen Z, et al. Passive sensing around the corner using spatial coherence. Nat Commun, 2018, 9: 3629

10 Zhu X, Yu J, Chen Y, et al. Experimental synthesis of random light sources with circular coherence by digital micro-mirror device. Appl Phys Lett, 2020, 117: 121102

11 Cao H, Chriki R, Bittner S, et al. Complex lasers with controllable coherence. Nat Rev Phys, 2019, 1: 156-168

12 Li Z, Liu W, Cheng H, et al. Few-layer metasurfaces with arbitrary scattering properties. Sci China-Phys Mech Astron, 2020, 63: 284202 